\title{
REGULARIZED 3D FUNCTIONAL REGRESSION FOR BRAIN IMAGE DATA VIA HAAR WAVELETS (SUPPLEMENTARY MATERIAL)
}

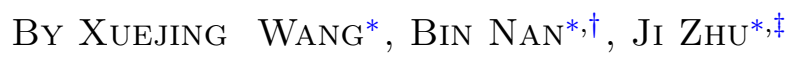 \\ Robert Koeppe* \\ University of Michigan
}

\section{APPENDIX A: THEORETICAL RESULTS}

We provide non-asymptotic theoretical properties of the proposed approach following the calculation of Bickel, Ritov and Tsybakov (2009). Though the results are described using the 1D notation, they hold exactly for the $3 \mathrm{D}$ case by viewing variable $t$ as a $3 \mathrm{D}$ variable.

Considering the Haar wavelet representation of $\beta$ with approximation error as in (2.2) in the paper, if we assume only a few elements of $\eta$ are nonzero, we can define $A_{\eta}=\left\{j: \eta_{j} \neq 0, j \in\{1, \cdots, p\}\right\}$. We further denote the cardinality of $A_{\eta}$ by $\left|A_{\eta}\right|$, which characterizes the sparsity of $\eta$. One common feature for the Lasso and the Dantzig selector is that, for the residual vector $\delta=\hat{\eta}-\eta$, the following relation holds with high probability:

$$
\left\|\delta_{A_{\eta}^{c}}\right\|_{1} \leq k_{0}\left\|\delta_{A_{\eta}}\right\|_{1}
$$

where $\delta_{A_{\eta}}$ is the subvector of $\delta$ consisting of any element of $\delta$ whose index is in $A_{\eta}$, and the constant $k_{0}$ is a positive number: $k_{0}=1$ for the Dantzig selector and $k_{0}=3$ for the Lasso (see Bickel, Ritov and Tsybakov, 2009). To achieve the oracle inequalities for the proposed estimator, we assume the same restricted eigenvalue assumption of Bickel, Ritov and Tsybakov (2009) on the Gram matrix $\frac{1}{n} C^{T} C$, which is a relaxation of the usual positive definiteness requirement and is given below.

Assumption . (Restricted Eigenvalue assumption $R E\left(s, k_{0}\right)$ ) For some integer $1 \leq s \leq p$ and $k_{0}>0$, the following holds:

$$
\kappa\left(s, k_{0}\right)=\min _{A_{\eta} \subseteq\{1, \cdots, p\}:\left|A_{\eta}\right| \leq s} \min _{\delta \neq 0:\left\|\delta_{A_{\eta}^{c}}\right\|_{1} \leq k_{0}\left\|\delta_{A_{\eta}}\right\|_{1}} \frac{\|C \delta\|_{2}}{\sqrt{n} \| \delta_{A_{\eta} \|_{2}}}>0 .
$$

\footnotetext{
${ }^{*}$ Supported in part by NIH grant R01-AG036802.

${ }^{\dagger}$ Supported in part by NSF Grant DMS-1007590.

${ }^{\ddagger}$ Supported in part by NSF Grant DMS-0748389.
} 
We assume that for a given finite wavelet decomposition with resolution $J$ and corresponding total number of basis functions $p$, all the diagonal elements of the matrix $\frac{C^{T} C}{n}$ be equal to 1 , which is achieved by scaling each column of $C$ by its root mean square. Then we have Theorem 1 and Theorem 2 .

THEOREM 1. Assume that $\epsilon_{i}$ in model (1.1) follows a normal distribution $N\left(0, \sigma_{1}^{2}\right)$ with $\sigma_{1}^{2}>0$. Suppose that $\eta$ is an $s$-sparse vector, where $1 \leq s \leq p$. Suppose that the assumption $R E\left(s, k_{0}\right)$ is satisfied with $k_{0}=3+4 / \theta$, for some $\theta>0$. Let $\hat{\eta}$ be the Lasso solution given in (2.5) with $\lambda=a \sigma_{1} \sqrt{\frac{\log p}{n}}$, $a>2 \sqrt{2}$. Then with probability at least $1-p^{1-a^{2} / 8}$, we have

$$
\begin{aligned}
& \left\|\int_{0}^{T} X(t) \hat{\beta}(t) d t-\int_{0}^{T} X(t) \beta(t) d t\right\|_{n}^{2} \\
& (\text { A.1 }) \leq(1+\theta) \inf _{\eta \in \mathbb{R}^{p}:\left|A_{\eta}\right| \leq s}\left\{\left\|\int_{0}^{T} X(t) e(t) d t\right\|_{n}^{2}+\frac{D(\theta) a^{2} \sigma_{1}^{2}}{\kappa^{2}}\left(\frac{s \log p}{n}\right)\right\},
\end{aligned}
$$

where $\kappa=\kappa(s, 3+4 / \theta)$ and $D(\theta)$ is a positive constant depending on $\theta$; and for this given $p$-dimensional basis, let $\omega=\sup _{t}|e(t)|$ and suppose that there exists an $M<\infty$ such that $\int\left|X_{i}(t)\right| d t \leq M$, then we have

$$
|\hat{\beta}(t)-\beta(t)| \leq \gamma(t)(4+4 / \theta)\left\{\frac{4 a s \sigma_{1} \sqrt{\frac{\log p}{n}}+2 \kappa \sqrt{s} M \omega}{\kappa^{2}}\right\}+\omega
$$

where

$$
\gamma(t)=\sum_{j=1}^{p}\left|\frac{b_{j}(t)}{\sqrt{\frac{1}{n} \sum_{i=1}^{n} C_{i j}^{2}}}\right|
$$

and $b_{j}(t)$ is the $j$-th basis function in $B(t)$.

Proof. Let $\hat{\eta}$ be the lasso solution given in (2.5) with $\lambda=a \sigma_{1} \sqrt{\frac{\log p}{n}}$, $a>2 \sqrt{2}$. In order to prove Theorem 1 , we need the following Lemma 1 , which is modified from Lemma B.1 of Bickel, Ritov and Tsybakov (2009). The proof of Lemma 1 follows similarly and thus is omitted.

Lemma 1. Assume the same assumptions as in Theorem 1, then with 
probability at least $1-p^{1-a^{2} / 8}$ we have

$$
\begin{aligned}
& \| \int_{0}^{T} X(t) \hat{\beta}(t) d t-\int_{0}^{T} X(t) \beta(t) d t\left\|_{n}^{2}+\lambda\right\| \hat{\eta}-\eta \|_{1} \\
& \leq\left\|\int_{0}^{T} X(t) e(t) d t\right\|_{n}^{2}+4 \lambda \sum_{j \in A_{\eta}}\left|\hat{\eta}_{j}-\eta_{j}\right| \\
& \leq\left\|\int_{0}^{T} X(t) e(t) d t\right\|_{n}^{2}+4 \lambda \sqrt{s} \sqrt{\sum_{j \in A_{\eta}}\left|\hat{\eta}_{j}-\eta_{j}\right|^{2}}
\end{aligned}
$$

where $e(t)$ is the approximation error in (2.2) and $\|\cdot\|_{n}$ is the empirical norm, which is defined as $\|g\|_{n}=\sqrt{\frac{1}{n} \sum_{i=1}^{n} g_{i}^{2}}$

The result in (A.1) is obtained immediately by applying Theorem 5.1 in Bickel, Ritov and Tsybakov (2009). Now we only need to prove (A.2).

By assumption $R E(s, 3+4 / \theta)$, we have

$$
\begin{aligned}
\kappa^{2}\left\|\delta_{A_{\eta}}\right\|_{2}^{2} & \leq \frac{1}{n}\|C \delta\|_{2}^{2} \\
& =\frac{1}{n}(\hat{\eta}-\eta)^{T} C^{T} C(\hat{\eta}-\eta) \\
& =\|C \hat{\eta}-C \eta\|_{n}^{2} \\
& \leq\left(\left\|C \hat{\eta}-\int_{0}^{T} X(t) \beta(t) d t\right\|_{n}+\left\|\int_{0}^{T} X(t) e(t) d t\right\|_{n}\right)^{2} .
\end{aligned}
$$

Lemma 1 implies,

$$
\begin{aligned}
\left\|\int_{0}^{T} X(t) \hat{\beta}(t) d t-\int_{0}^{T} X(t) \beta(t) d t\right\|_{n}^{2} & =\left\|C \hat{\eta}-\int_{0}^{T} X(t) \beta(t) d t\right\|_{n}^{2} \\
& \leq\left\|\int_{0}^{T} X(t) e(t) d t\right\|_{n}^{2}+4 \lambda \sqrt{s}\left\|\delta_{A_{\eta}}\right\|_{2} .
\end{aligned}
$$

Combining (A.3) with (A.4), we find

$$
\begin{aligned}
\kappa\left\|\delta_{A_{\eta}}\right\|_{2} & \leq\left\|C \hat{\eta}-\int_{0}^{T} X(t) \beta(t) d t\right\|_{n}+\left\|\int_{0}^{T} X(t) e(t) d t\right\|_{n} \\
\text { A.5) } & \leq \sqrt{\left\|\int_{0}^{T} X(t) e(t) d t\right\|_{n}^{2}+4 \lambda \sqrt{s}\left\|\delta_{A_{\eta}}\right\|_{2}}+\left\|\int_{0}^{T} X(t) e(t) d t\right\|_{n} .
\end{aligned}
$$


Subtracting the second term of (A.5) from both sides, and then squaring both bides, we have

$$
\begin{aligned}
\left(\kappa\left\|\delta_{A_{\eta}}\right\|_{2}\right. & \left.-\left\|\int_{0}^{T} X(t) e(t) d t\right\|_{n}\right)^{2} \\
& \leq\left\|\int_{0}^{T} X(t) e(t) d t\right\|_{n}^{2}+4 \lambda \sqrt{s}\left\|\delta_{A_{\eta}}\right\|_{2} .
\end{aligned}
$$

To solve the quadratic inequality for $\left\|\delta_{A_{\eta}}\right\|_{2}$, we first need to expand the left side of (A.6), which yields

$$
\kappa^{2}\left\|\delta_{A_{\eta}}\right\|_{2}^{2}-2 \kappa\left\|\delta_{A_{\eta}}\right\|_{2}\left\|\int_{0}^{T} X(t) e(t) d t\right\|_{n} \leq 4 \lambda \sqrt{s}\left\|\delta_{A_{\eta}}\right\|_{2} .
$$

This implies

$$
\begin{aligned}
\left\|\delta_{A_{\eta}}\right\|_{2} & \leq \frac{4 \lambda \sqrt{s}+2 \kappa\left\|\int_{0}^{T} X(t) e(t) d t\right\|_{n}}{\kappa^{2}} \\
& \leq \frac{4 \lambda \sqrt{s}+2 \kappa M \omega}{\kappa^{2}}
\end{aligned}
$$

We also observed that the following relations hold with $k_{0}=3+4 / \theta$ :

$$
\|\delta\|_{1}=\left\|\delta_{A_{\eta}}\right\|_{1}+\left\|\delta_{A_{\eta}^{c}}\right\|_{1} \leq\left(1+k_{0}\right)\left\|\delta_{A_{\eta}}\right\|_{1} \leq\left(1+k_{0}\right) \sqrt{s}\left\|\delta_{A_{\eta}}\right\|_{2} .
$$

Then we have,

$$
\|\delta\|_{1} \leq(4+4 / \theta) \sqrt{s}\left\{\frac{4 \lambda \sqrt{s}+2 \kappa M \omega}{\kappa^{2}}\right\}
$$

Now let $D$ be a diagonal matrix with $\sqrt{\sum_{i=1}^{n} C_{i j}^{2}}$ as the $j$ th diagonal element. We can then rewrite model (2.4) as

$$
Y=\beta_{0}+C \eta+\epsilon^{*}=\beta_{0}+C\left(\sqrt{n} D^{-1}\right)\left(\frac{1}{\sqrt{n}} D\right) \eta+\epsilon^{*}=\beta_{0}+\tilde{C} \tilde{\eta}+\epsilon^{*}
$$

where $\tilde{C}=C\left(\sqrt{n} D^{-1}\right)$ and $\tilde{\eta}=\left(\frac{1}{\sqrt{n}} D\right) \eta$. Then the diagonal elements of 
the matrix $\frac{\tilde{C}^{T} \tilde{C}}{n}$ are equal to 1 . Therefore, we obtain

$$
\begin{aligned}
|\hat{\beta}(t)-\beta(t)| & \leq\left|\hat{\beta}(t)-B(t)^{T} \eta\right|+|e(t)| \\
& =\left|B(t)^{T} \hat{\eta}-B(t)^{T} \eta\right|+|e(t)| \\
& \leq \sqrt{n}\left\|B(t)^{T} D^{-1}\right\|_{1}\|\hat{\tilde{\eta}}-\tilde{\eta}\|_{1}+\omega \\
& =\gamma(t)\|\hat{\tilde{\eta}}-\tilde{\eta}\|_{1}+\omega \\
& \leq \gamma(t)(4+4 / \theta)\left\{\frac{4 a s \sigma_{1} \sqrt{\frac{\log p}{n}}+2 \kappa \sqrt{s} M \omega}{\kappa^{2}}\right\}+\omega
\end{aligned}
$$

where $\tilde{\hat{\eta}}=\left(\frac{1}{\sqrt{n}} D\right) \hat{\eta}$ and $\gamma(t)=\sqrt{n}\left\|B(t)^{T} D^{-1}\right\|_{1}=\sum_{j=1}^{p}\left|\frac{b_{j}(t)}{\sqrt{\frac{1}{n} \sum_{i=1}^{n} C_{i j}^{2}}}\right|$.

If we further assume that $\epsilon_{i}^{*} \sim N\left(0, \sigma_{2}^{2}\right)$ in model $(2.4)$, we have the following result.

Theorem 2. Assume that $\epsilon_{i}^{*} \sim N\left(0, \sigma_{2}^{2}\right)$ with $\sigma_{2}^{2}>0$. The model (2.4) reduces to a linear regression problem. Suppose that $\eta$ is an s-sparse vector with $1 \leq s \leq p$. Let $\hat{\eta}$ be the corresponding Lasso solution with $\lambda=$ $a \sigma_{2} \sqrt{\frac{\log p}{n}}$, and $a>2 \sqrt{2}$. Also suppose there exists an $M<\infty$ such that $\int\left|X_{i}(t)\right| d t \leq M$. Then under assumption $R E(s, 3)$, with probability at least $1-p^{1-a^{2} / 8}$, we have

$$
\begin{gathered}
\left\|\int X(t) \hat{\beta}(t) d t-\int X(t) \beta(t) d t\right\|_{n} \leq \frac{4 a \sigma_{2}}{\kappa} \sqrt{\frac{s \log p}{n}}+M \omega \\
|\hat{\beta}(t)-\beta(t)| \leq \gamma(t) \frac{16 a s \sigma_{2}}{\kappa^{2}} \sqrt{\frac{\log p}{n}}+\omega
\end{gathered}
$$

where $\kappa=\kappa(s, 3)$.

Proof. If we assume that $\epsilon_{i}^{*} \sim N\left(0, \sigma_{2}^{2}\right)$ in model (2.4), then this model is the special case in Section 6 of Bickel, Ritov and Tsybakov (2009). Let $\hat{\eta}$ be the corresponding lasso solution with $\lambda=a \sigma_{2} \sqrt{\frac{\log p}{n}}, a>2 \sqrt{2}$, then Lemma 2 here is obtained directly from their Theorem 6.2.

Lemma 2. Assume the same assumptions as in Theorem 2. Then under assumption $R E(s, 3)$, with the probability at least $1-p^{1-a^{2} / 8}$ we have

$$
\|\hat{\eta}-\eta\|_{1} \leq \frac{16 a s \sigma_{2}}{\kappa^{2}} \sqrt{\frac{\log p}{n}}
$$




$$
\|C(\hat{\eta}-\eta)\|_{2}^{2} \leq \frac{16 a^{2} s \sigma_{2}^{2}}{\kappa^{2}} \log p
$$

where $\kappa=\kappa(s, 3)$.

It follows from (A.8) that

$$
\|C(\hat{\eta}-\eta)\|_{n}=\frac{1}{\sqrt{n}}\|C(\hat{\eta}-\eta)\|_{2} \leq \frac{4 a \sigma_{2}}{\kappa} \sqrt{\frac{s \log p}{n}} .
$$

We then have

$$
\begin{aligned}
\left\|\int_{0}^{T} X(t) \hat{\beta}(t) d t-\int_{0}^{T} X(t) \beta(t) d t\right\|_{n} & \leq\|C(\hat{\eta}-\eta)\|_{n}+\left\|\int_{0}^{T} X(t) e(t) d t\right\|_{n} \\
& =\|\tilde{C}(\hat{\tilde{\eta}}-\tilde{\eta})\|_{n}+\left\|\int_{0}^{T} X(t) e(t) d t\right\|_{n} \\
& \leq\|\tilde{C}(\hat{\tilde{\eta}}-\tilde{\eta})\|_{n}+M \omega \\
& \leq \frac{4 a \sigma_{2}}{\kappa} \sqrt{\frac{s \log p}{n}}+M \omega .
\end{aligned}
$$

By (A.7), we have

$$
\|\hat{\tilde{\eta}}-\tilde{\eta}\|_{1} \leq \frac{16 a s \sigma_{2}}{\kappa^{2}} \sqrt{\frac{\log p}{n}}
$$

Therefore, we obtain

$$
\begin{aligned}
|\hat{\beta}(t)-\beta(t)| & \leq\left|\hat{\beta}(t)-B(t)^{T} \eta\right|+|e(t)| \\
& =\left|B(t)^{T} \hat{\eta}-B(t)^{T} \eta\right|+|e(t)| \\
& \leq \sqrt{n}\left\|B(t)^{T} D^{-1}\right\|_{1}\|\tilde{\tilde{\eta}}-\tilde{\eta}\|_{1}+\omega \\
& =\gamma(t)\|\hat{\tilde{\eta}}-\tilde{\eta}\|_{1}+\omega \\
& \leq \gamma(t) \frac{16 a s \sigma_{2}}{\kappa^{2}} \sqrt{\frac{\log p}{n}}+\omega .
\end{aligned}
$$

\section{APPENDIX B: DETAILED EXPLANATION FOR CHOOSING HAAR WAVELETS}

The methods developed in this article can yield a sparse estimation of $\beta(t)$ because when the true $\beta(t)$ is zero in many regions, the Haar wavelet 
coefficients of $\beta(t)$ are usually sparse too. Therefore, we can apply the variable selection methods, such as the lasso, to select the nonzero Haar wavelet coefficients.

We now illustrate this feature via a simple example. Suppose that $\beta(t)$ is a $1 \mathrm{D}$ signal and collected at 8 time points. $\beta(t)=\left[\beta\left(t_{1}\right), \ldots, \beta\left(t_{8}\right)\right]=$ $[0,0,4,6,5,5,0,0]$. Applying level-1 Haar wavelet transform to $\beta(t)$, we obtain the wavelet coefficient vector $c_{1}$. The Haar transformation matrix for this case is shown below:

$$
\begin{aligned}
& H_{1}=\frac{1}{\sqrt{2}}\left[\begin{array}{cccccccc}
1 & 1 & 0 & 0 & 0 & 0 & 0 & 0 \\
0 & 0 & 1 & 1 & 0 & 0 & 0 & 0 \\
0 & 0 & 0 & 0 & 1 & 1 & 0 & 0 \\
0 & 0 & 0 & 0 & 0 & 0 & 1 & 1 \\
1 & -1 & 0 & 0 & 0 & 0 & 0 & 0 \\
0 & 0 & 1 & -1 & 0 & 0 & 0 & 0 \\
0 & 0 & 0 & 0 & 1 & -1 & 0 & 0 \\
0 & 0 & 0 & 0 & 0 & 0 & 1 & -1
\end{array}\right], \\
& \beta H_{1}^{T}=c_{1}=[0,5 \sqrt{2}, 5 \sqrt{2}, 0,0,-\sqrt{2}, 0,0],
\end{aligned}
$$

where $\frac{1}{\sqrt{2}}$ is the scaling factor and $c_{1}$ is the wavelet coefficient vector. Conversely, we have this relationship: $c_{1} H_{1}=\beta$. The first four coefficients in $c_{1}$ corresponding to the first four rows of $H_{1}$ are called approximation coefficients $\left(a_{1}\right)$, and the last four coefficients in $c_{1}$ corresponding to the last four rows of $H_{1}$ are called detail coefficients $\left(d_{1}\right)$. The first, third and last elements of $d_{1}$ are zero, meaning that there are no changes between the first two, third two and last two elements of $\beta(t)$. The first and last elements of $a_{1}$ are zero, indicating that the averages of the first and the last two elements of $\beta(t)$ are zero. This example shows that zeros in the detail coefficients do not always indicate zeros in the original signal, but no change between the two neighboring elements (for level-1 Haar wavelet decomposition). If the corresponding approximation coefficients are also zero, then some neighboring points are exactly zeros in the original signal. In this case, the first and the last elements of both $a_{1}$ and $d_{1}$ are zero, showing that the first and the last two elements of the original signal are zero.

The same feature holds for other levels Haar wavelet transform for $\beta(t)$. Let $H_{2}$ denote the level-2 Haar wavelet transform matrix, and $H_{3}$ denote the level-3 Haar wavelet transform matrix. We then have 


$$
\begin{aligned}
& H_{2}=\left[\begin{array}{cccccccc}
\frac{1}{2} & \frac{1}{2} & \frac{1}{2} & \frac{1}{2} & 0 & 0 & 0 & 0 \\
0 & 0 & 0 & 0 & \frac{1}{2} & \frac{1}{2} & \frac{1}{2} & \frac{1}{2} \\
\frac{1}{2} & \frac{1}{2} & -\frac{1}{2} & -\frac{1}{2} & 0 & 0 & 0 & 0 \\
0 & 0 & 0 & 0 & \frac{1}{2} & \frac{1}{2} & -\frac{1}{2} & -\frac{1}{2} \\
\frac{1}{\sqrt{2}} & -\frac{1}{\sqrt{2}} & 0 & 0 & 0 & 0 & 0 & 0 \\
0 & 0 & \frac{1}{\sqrt{2}} & -\frac{1}{\sqrt{2}} & 0 & 0 & 0 & 0 \\
0 & 0 & 0 & 0 & \frac{1}{\sqrt{2}} & -\frac{1}{\sqrt{2}} & 0 & 0 \\
0 & 0 & 0 & 0 & 0 & 0 & \frac{1}{\sqrt{2}} & -\frac{1}{\sqrt{2}}
\end{array}\right], \\
& \beta H_{2}^{T}=c_{2}=[5,5,-5,5,0,-\sqrt{2}, 0,0],
\end{aligned}
$$

and

$$
\begin{aligned}
& H_{3}=\left[\begin{array}{cccccccc}
\frac{1}{2 \sqrt{2}} & \frac{1}{2 \sqrt{2}} & \frac{1}{2 \sqrt{2}} & \frac{1}{2 \sqrt{2}} & \frac{1}{2 \sqrt{2}} & \frac{1}{2 \sqrt{2}} & \frac{1}{2 \sqrt{2}} & \frac{1}{2 \sqrt{2}} \\
\frac{1}{2 \sqrt{2}} & \frac{1}{2 \sqrt{2}} & \frac{1}{2 \sqrt{2}} & \frac{1}{2 \sqrt{2}} & -\frac{1}{2 \sqrt{2}} & -\frac{1}{2 \sqrt{2}} & -\frac{1}{2 \sqrt{2}} & -\frac{1}{2 \sqrt{2}} \\
\frac{1}{2} & \frac{1}{2} & -\frac{1}{2} & -\frac{1}{2} & 0 & 0 & 0 & 0 \\
0 & 0 & 0 & 0 & \frac{1}{2} & \frac{1}{2} & -\frac{1}{2} & -\frac{1}{2} \\
\frac{1}{\sqrt{2}} & -\frac{1}{\sqrt{2}} & 0 & 0 & 0 & 0 & 0 & 0 \\
0 & 0 & \frac{1}{\sqrt{2}} & -\frac{1}{\sqrt{2}} & 0 & 0 & 0 & 0 \\
0 & 0 & 0 & 0 & \frac{1}{\sqrt{2}} & -\frac{1}{\sqrt{2}} & 0 & 0 \\
0 & 0 & 0 & 0 & 0 & 0 & \frac{1}{\sqrt{2}} & -\frac{1}{\sqrt{2}}
\end{array}\right], \\
& \beta H_{3}^{T}=c_{3}=[5,0,-5,5,0,-\sqrt{2}, 0,0] .
\end{aligned}
$$

By observing the structures of different levels Haar wavelet transform, we find that the numbers of nonzero elements in the wavelet coefficient vectors are different. It is not surprising to see that $\hat{\beta}(t)$ is often piece-wise constant after applying the lasso method for the selection of important elements of wavelet coefficients because small detail coefficients are set to zero. Moreover, higher-level Haar wavelet transform tends to give us piecewise constant solutions on a coarser scale. Considering all of the above, it would be natural to assume that there exists an optimal wavelet decomposition level for a given signal in terms of measures of prediction accuracy in the regression setting. Therefore, we set it as an additional tuning parameter.

\section{REFERENCES}

Bickel, P., Ritov, Y. and Tsybakov, A. (2009). Simultaneous analysis of Lasso and Dantzig Selector. Annals of Statistics 4 1705-1732. 
Department of Biostatistics

UNIVERSITY OF MICHIGAN

1415 Washington Heights

Ann Arbor, Michigan 48109-2029

E-MAIL: xuejwang@umich.edu bnan@umich.edu
Department of Statistics

UNIVERSITY OF MICHIGAN

1085 South University Ave

Ann Arbor, Michigan 48109-1107

E-MAIL: jizhu@umich.edu

DEPARTMENT OF RADiology

UNIVERSITY OF MiCHIGAN

1500 E. Medical Center Drive

Ann Arbor, Michigan 48109-5030

E-MAIL: koeppe@umich.edu 